\title{
FIELD OF A CHARGED PARTICLE IN THE PRESENCE OF SCALAR MESON FIELDS IN GENERAL RELATIVITY
}

\author{
D. R. K. REDDY and V. U. M. RAO'
}

(Received 3 December 1981; revised 5 April 1982)

\begin{abstract}
Field equations for coupled gravitational and zero mass scalar fields in the presence of a point charge are obtained with the aid of a static spherically symmetric conformally flat metric. A closed form exact solution of the field equations is presented which may be considered as describing the field of a charged particle at the origin surrounded by the scalar meson field in a conformally flat space-time.
\end{abstract}

\section{Introduction}

The study of scalar meson fields in general relativity has drawn the attention of many workers. Bergmann and Leipnik [1], Buchdahl [3], Bramhachary [2], Stephenson [13], Penney [6, 7], Gautreau [4], Rao et al . [9], Roy and Datta [11], Roy and Rao [12] are some of the authors who have investigated various aspects of the problem.

Recently, Penney [8] and Gurses [5] have obtained exact solutions for massless scalar meson fields with a conformally flat metric while Ray [10] has given a complete set of exact solutions for both massive and massless scalar mesons in a conformally flat space-time. Here we have considered the energy-momentum tensor due to a source-free electromagnetic field and have obtained an exact solution of the Einstein-Maxwell-massless scalar field equations for a static charged point mass with the aid of a conformally flat metric.

\footnotetext{
${ }^{1}$ Department of Applied Mathematics, Andhra University, Waitair-530 003, India.

C Copyright Australian Mathematical Society 1983
} 


\section{Field equations}

The general relativistic field equations for the interacting source-free electromagnetic and zero mass scalar (meson) fields are given by

$$
R_{i j}-\frac{1}{2} g_{i j} R=-8 \pi\left(E_{i j}+T_{i j}\right),
$$

where

$$
E_{i j}=\frac{1}{4 \pi}\left(F_{i l} F_{j}^{\prime}-\frac{1}{4} g_{\imath j} F_{k l} F^{k l}\right)
$$

is the energy momentum tensor due to the source-free electromagnetic field and

$$
T_{i j}=\frac{1}{4 \pi}\left[\phi_{, i} \phi_{, j}-\frac{1}{2} g_{i j} \phi_{, k} \phi^{k k}\right]
$$

is the energy momentum for the zero mass scalar field. Here $F_{i}$ satisfies the Maxwell field equations

$$
\left.\begin{array}{l}
F_{\imath j}=A_{i, j}-A_{j, i}, \\
F_{: j}^{i j}=0,
\end{array}\right\}
$$

$A_{t}$ being the four potential and the scalar field $\phi$ satisfies the wave equation

$$
\phi_{; k}^{, k}=0 \text {. }
$$

Here a comma and semicolon denote partial and covariant derivatives respectively.

In the following section, we solve the field equations (1) to (5) for a static spherically symmetric conformally flat metric

$$
d s^{2}=e^{\alpha}\left(d r^{2}+r^{2} d \sigma^{2}+r^{2} \sin ^{2} \sigma d \Phi^{2}-d t^{2}\right)
$$

where $\alpha$ is a function of $r$ alone. The spherical symmetry assumed implies that the scalar meson field $\phi$ shares the same symmetry.

From (2), (4) and (6) the non-vanishing components of the energy momentum tensor for the electrostatic case are found to be

$$
E_{1}^{1}=-E_{2}^{2}=-E_{3}^{3}=E_{4}^{4}=-\frac{1}{8 \pi} e^{-2 \alpha} \frac{q^{2}}{r^{4}},
$$

where $q$ is a constant which is identified with the electric charge.

Taking $\phi$ as a functioin of $r$ only and using (3), (6) and (7) in (1) and (5) the coupled Einstein-Maxwell-massless scalar field equations for a charged point mass can be written explicitly as

$$
\frac{3}{4} \alpha^{2}+\frac{2 \alpha^{\prime}}{r}=-\frac{q^{2}}{r^{4}} e^{-\alpha}+\phi^{\prime 2}
$$




$$
\begin{gathered}
\alpha^{\prime \prime}+\frac{\alpha^{2}}{4}+\frac{\alpha^{\prime}}{r}=\frac{q^{2}}{r^{4}} e^{-\alpha}-\phi^{\prime 2}, \\
\alpha^{\prime \prime}+\frac{\alpha^{\prime 2}}{4}+\frac{2 \alpha^{\prime}}{r}=-\frac{q^{2}}{r^{4}} e^{-\alpha}-\phi^{\prime 2}, \\
\phi^{\prime \prime}+\phi^{\prime}\left(\alpha^{\prime}+\frac{2}{r}\right)=0 .
\end{gathered}
$$

Here a superscript prime indicates differentiation with respect to $r$.

\section{Solutions of the field equations}

It can be easily verified that when the charge $q=0$, the field equations (8) to (11) yield a solution which describes an empty flat space-time in Einstein's theory with $\phi=$ constant. The constant may also be set equal to zero. That is a static spherically symmetric conformally flat solution of the Einstein field equations in the presence of zero mass scalar fields describes simply the empty flat space-time of general relativity.

When the massless scalar field $\phi \neq 0$ and when the charge $q \neq 0$, we solve the field equations (8) to (11). Since there are four field equations with only two unknowns, the derived solution is substituted in the equations and found to satisfy all four.

It is a simple matter to see that the field equations (9) to (11) reduce to

$$
e^{\alpha} \alpha^{\prime}=-2 q^{2} / r^{3}
$$

and

$$
r^{2} e^{\alpha} \phi^{\prime}=C_{1}
$$

which in turn give us

$$
\begin{aligned}
& e^{\alpha}=C_{2}+q^{2} / r^{2}, \quad C_{2} \neq 0, \\
& \phi=\frac{C_{1}}{q \sqrt{-C_{2}}} \frac{1}{2} \log \left|\frac{1+u}{1-u}\right|+\phi_{0},
\end{aligned}
$$

where $u=\left(\sqrt{-C_{2}} / q\right) r$ and $C_{1}, C_{2}$ and $\phi_{0}$ are constants of integration. It is seen that the solution (13) satisfies each of the field equations (8) to (11) provided the constants $C_{1}, C_{2}$ and $q^{2}$ are related by

$$
C_{1}^{2}+3 C_{2} q^{2}=0
$$

and $\phi_{0}$ can be set equal to zero. 
It can be seen that equation (14) together with $C_{1}^{2}>0$ and $q^{2}>0$ implies $C_{2}<0$. Therefore $\left(q \sqrt{-C_{2}}\right)$ in (13) becomes real. Thus equations (6) and (13) along with (14) constitute an exact static spherically symmetric conformally flat solution of the Einstein-Maxwell-massless scalar field equations.

Physically the solution (13) along with (14) may be looked upon as describing the gravitational field of a charged particle at the origin surrounded by the field of a zero-mass meson in a conformally flat space-time. It can be seen that the solution (13) is asymptotically flat and has a singularity at $u=1$. It is interesting to note from (14) that the constant $C_{1}=0$, implies $q=0\left(C_{2} \neq 0\right)$ and the solution goes over to the empty flat space-time of Einstein's theory with $\phi=0$.

\section{Conclusions}

The need for exact solutions in the general theory of relativity is well known. Here we obtained a closed form exact static spherically symmetric conformally flat solution of the coupled Einstein-Maxwell-massless scalar field equations corresponding to a charged mass point at the origin surrounded by the field of a zero mass meson. It is heartening to realise that we are able to obtain an analytic solution of a problem with three fields coupled non-linearly. The solution (13) will play a physically significant role in studies of the interaction of electromagnetic and scalar meson fields in a conformally flat space-time. It may be mentioned here that the study of interacting electromagnetic and meson fields is of considerable interest in the field of high energy physics.

\section{Acknowledgements}

The authors thank the referee for his useful comments. Also one of the authors (V. U. M. Rao) is grateful to the CSIR, India, for financial assistance.

\section{References}

[1] O. Bergmann and R. Leipnik, "Space-time structure of a static spherically symmetric scalar field", Phys. Rev. 107 (1957), 1157-1161.

[2] H. Bramhachary, "A solution of the combined gravitational and meson field equations in general relativity", Progr. Theoret. Phys. 23 (1960), 749-750.

[3] H. A. Buchdahl, "Reciprocal static metrics and scalar fields in the general theory of relativity", Phys. Rev. 115 (1959), 1325-1328. 
[4] R. Gautreau, "Coupled Weyl gravitational and zero-rest-mass scalar fields", Nuovo Cimento B 62 (1969), 360-370.

[5] M. Gurses, "Conformally flat solutions of Einstein-massless-scalar field equations", Phys. Rev. D 15 (1977), 2731-2733.

[6] R. Penney, “Axially symmetric zero-mass meson solutions of Einstein equations", Phys. Rev. 174 (1968), 1578-1579.

[7] R. Penney, "Generalization of the Reissner-Nordstrom solution to the Einstein field equations", Phys. Rev. 182 (1969), 1383-1384.

[8] R. Penney, "Conformally flat zero-mass meson solutions of Einstein equations", Phys. Rev. D 14 (1975), 910-911.

[9] J. R. Rao, A. R. Roy and R. N. Tiwari, "A class of exact solutions for coupled electromagnetic and scalar fields for Einstein-Rosen metric", Ann. Physics 69 (1972), 473-486.

[10] D. Ray, "Scalar meson fields in a conformally flat space", J. Math. Phys. 18 (1977), 1899-1901.

[11] A. R. Roy and C. R. Datta, "Time dependent axially symmetric solutions of the EinsteinMaxwell-Yukawa fields", Commun. Math. Phys. 29 (1973), 285-292.

[12] A. R. Roy and J. R. Rao, "Non-existence of axially symmetric massive scalar fields", Comm. Math. Phys. 27 (1972), 162-166.

[13] G. Stephenson, "A static spherically symmetric solution of the Einstein-Maxwell-Yukawa field equations”, Proc. Cambridge Phlos. Soc. 58 (1962), 521-526. 